\title{
ROMPERE IL TABÙ: IL TEMA DELL'ABBANDONO DEI FIGLI IN UNA DONNA DI SIBILLA ALERAMO E LA FIGLIA OSCURA DI ELENA FERRANTE ${ }^{1}$
}

\author{
Sanja Kobilj Cuic ${ }^{2}$
}

\begin{abstract}
Rompere il tabù: Il tema dell'abbandono dei figli in Una donna di Sibilla Aleramo e La filgia oscura di Elena Ferrante

Riassunto: Questo articolo esamina la maternità rappresentata in due testi, Una donna di Sibilla Aleramo e La figlia osura di Elena Ferrante. L'accento è sul tema dell'ambivalenza materna e sul tema dell'abbandono dei figli da parte delle madri considerato tuttora uno dei tabù nella società e nella letteratura italiane. Attraverso l'analisi di questi due romanzi, si cerca di capire quali siano i motivi emotivi e sociali che portano le madri protagoniste a cercare i propri spazi. Il metodo teorico si basa sul lavoro del gruppo Diotima sull'ambivalenza della maternità.
\end{abstract}

Parole chiave: Scrittrici italiane, maternità, abbandono dei figli, ambivalenza materna, Sibilla Aleramo, Elena Ferrante.

\section{Breaking the Taboo: The Theme of Abandonment of Children}

Abstract: This article examines motherhood represented in some of the most important novels of Italian female literature. The focus is on the topic of maternal ambivalence and the on theme of abandonment of a child considered one of the taboos in Italian literature and society. Through a close reading of novels Una donna written by Sibilla Aleremo and La figlia oscura and I giorni dell'abbandono by Elena Ferrante, the article analyses the emotional and social motives that bring the women to search for their own freedom and re-establish their own identity. The search for a non-patriarchal motherhood is made possible through the work of Italian theorist gathered around the Italian feminist group of Diotima that through different seminars discuss the exception of a mother and explore the ambivalence of the maternal. Although the changes in better are visible, the narrative of women needs more exploring of the voice of the mother but also a larger autonomy in expressing the dark sides of maternity that still lack of presentation.

Key words: Italian women writers, motherhood, abandonment of a child, maternal ambivalence, Sibilla Aleramo, Elena Ferrante.

\section{Premessa}

${ }^{1}$ Data di ricezione: $2 / 08 / 2015$.

Data di accettazione: 20/09/2015.

${ }^{2}$ Docente, Filološki fakultet, Dipartimento di Italianistica, Università di Banja Luka, Banja Luka, Bosnia ed Erzegovina; $₫$ sanja.zeno@gmail.com. 
L'abbandono dei figli da parte della madre rappresenta tutt'oggi uno dei temi letterari considerati tabù largamente presenti sia nella letteratura che nella società italiana. Da quando Ibsen pubblicò Casa di bambola, (un evento letterario che ha suscitato un forte disagio e scandalo nella società) in cui la protagonista Nora abbandona i propri figli per ricostruire la propria identità. Ancora oggi, di questo tema si parla poco, anche perché si tende a non riflettere sulle motivazioni che spingono le protagoniste madri a compiere una scelta così radicale. Nonostante la maternità sia uno dei temi apparentemente più affrontati dal punto di vista editoriale e narrativo, le questioni legate alle sue ambivalenze come depressione, isolamento sociale, economico e emotivo delle madri sono poco affrontati. Le poche madri letterarie che osano ribellarsi a queste condizioni non hanno scelta o alternativa; essendo le voci femminili molto soffocate, esse finiscono la propria "ribellione" oppresse dai sensi di colpa, ma soprattutto dai giudizi esterni, quelli della società o quelli dei propri figli o altri membri della famiglia. Invece, la presenza delle madri letterarie che combattono il proprio senso di inadeguattezza prendendo spazio per sé e non limitandosi esclusivamente al ruolo di madre, è importante: raccontandosi e affrontando le vie delle viscere della maternità è possibile rimuovere barriere sociali che definiscono la maternità .

Lo scopo di questo intervento è dimostrare come sia stata affrontata l'ambivalenza della maternità in due testi narrativi che ruotano intorno al tema dell'abbandono dei figli da parte delle madri. Il tentativo è quello di riflettere sui motivi che spingono le madri letterarie a cercare spazi propri. L'arco del tempo dei testi analizzati è molto ampio: il romanzo ormai leggendario di Sibilla Aleramo Una donna è pubblicato nel 1906, mentre I giorni dell'abbandono e La figlia oscura di Elena Ferrante ${ }^{3}$ sono stati pubblicati rispettivamente nel 2002 e 2006. In questo articolo cercherò di analizzare quanto sia cambiata la situazione sociale ed emotiva delle madri letterarie, quanto cioè la loro voce è riuscita a liberarsi dalle costrizioni sociali a cui è stata tradizionalmente esposta.

\footnotetext{
3 Elena Ferrante, dopo il grande successo dei suoi romanzi, L'amore molesto (1992), I giorni dell'abbandono (2002), La figlia oscura (2006) e soprattutto della tetralogia L'amica geniale (L'amica geniale del 2011, Storia del nuovo cognome del 2012, Storia di chi fugge e di chi resta del 2013 e Storia della bambina perduta del 2014) rimane tuttora un fenomeno editoriale perché dell'identità della scrittrice non si sa niente. Esistono diverse ipotesi su chi si nasconde dietro lo pseudonimo di Elena Ferrante, di cui nessuna finora ha avuto la conferma.
} 
Il mio approccio intende essere inter-disciplinare. Saranno presi in considerazione cambiamenti sociali avvenuti nell'arco di un secolo insieme al lavoro teorico sull'ambivalenza materna svolto dalle teoriche legate al gruppo Diotima ${ }^{4}$ che hanno proseguito la ricerca francese e americana sul campo ${ }^{5}$.

\section{Una donna di Sibilla Aleramo}

Una donna è un romanzo difficilmente classificabile, perché "non è un romanzo tradizionale, non è un'autobiografia, non è un diario intimo, ma attraversa tutti questi generi" (Chemotti 2009: 31). Sibilla Aleramo, pseudonimo di Rina Faccio, usa diverse tecniche narrative per raccontare la storia di un matrimonio fallito e di un processo di crescita personale, che raggiunge il climax nella scelta di lasciare la casa, il marito e il figlio per guadagnare in tal modo la tanto desiderata libertà individuale.

Il romanzo scritto in prima persona segue la formazione della protagonista, che evolve da un'adolescente per la quale il centro dell'esistenza è rappresentato dal padre, attraverso il matrimonio con un ragazzo che la violenta, e la maternità vissuta come una "potenzialità naturale", fino alla presa di coscienza di sé ma anche della posizione della donna in una società maschilista. Quest'ultima porta la protagonista a mettere in dubbio il significato della maternità, ma anche a comprendere l'ipocrisia della società che depriva la donna della possibilità di ragionare da sé. La maternità viene comunemente rappresentata come un estremo sacrificio, che dovrà passare da madre in figlia, per creare una catena di donne schiave delle proprie emozioni:

\footnotetext{
${ }^{4}$ Diotima, la comunità filosofica italiana nasce presso l'università di Verona nel 1983, con l'intento di “essere donne e pensare filosoficamente". Le studiose di questo gruppo hanno organizzato nel passato "grandi seminari annuali" su diversi temi che riguardano l'essere donna, da cui sono nati i loro libri: Il pensiero della differenza sessuale, Mettere al mondo il mondo, Il cielo stellato dentro di noi, Oltre l'uguaglianza, La sapienza di partire da sé, Il profumo della maestra, Approfittare dell'assenza, L'ombra della madre.

${ }^{5}$ Le studiose di Diotima si sono accostate soprattutto al pensiero di Luce Irigaray, ma anche a quello di Helene Cixous, Nancy Chodorow, Adrienne Rich e molte altre. È importante nominare anche le studiose italiane che hanno fatto un enorme contributo sul tema del materno: Luisa Muraro, Silvia Vegetti Finzi, Marina Zancan.
} 
Perché nella maternità adoriamo il sacrificio? Donde è scesa a noi questa inumana idea dell'immolazione materna? Di madre in figlia, da secoli, si tramanda il servaggio. È una mostruosa catena. Tutte abbiamo, a un certo punto della vita, la coscienza di quel che fece pel nostro bene chi ci generò: e con la coscienza il rimorso di non aver compensato adeguatamente l'olocausto della persona diletta. Allora riversiamo sui nostri figli quanto non demmo alle madri, rinnegando noi stesse e offrendo un nuovo esempio di mortificazione, di annientamento.

Se una buona volta la fatale catena si spezzasse, e una madre non sopprimesse in sé la donna, e un figlio apprendesse dalla vita di lei un esempio di dignità?

Per quello che siamo, per la volontà di tramandare più nobile e più bella in essi la vita, devono esserci grati i figli, non perché, dopo averli ciecamente suscitati dal nulla, rinunziamo all'essere noi stessi. (Aleramo 2007: 145)

Dunque, solo spezzando la catena delle donne che passano ai figli lo spirito del sacrificio a loro volta ereditato dalle proprie madri, potrebbe cambiare il modo di vivere la maternità. La protagonista decide di lasciare il marito quando capisce che potrebbe perdere la propria identità tra persone con le quali non riesce a parlare di quello che prova:

Certe sere, tutti se n'andavano lasciandomi sola: portavo il bimbo a letto e poi mi affondavo in un seggiolone di paglia nel giardino. La cupa volta cosparsa di mondi silenziosi attraeva il mio sguardo magneticamente; ma il mistero dell'universo non mi tentava in quell'ore: un'angoscia umana, precisa, incalzante, mi possedeva intera; l'amarezza senza nome della mia solitudine, il vago timore di una morte possibile, prossima, lì tra quella gente ostile e straniera, senza aver lasciato traccia della mia anima [...]. Tanto spazio di cielo, ed io incatenata, curva sotto un giogo spietato, non capace più che di un lento pianto. (Aleramo 2007: 142) ${ }^{6}$

Insieme a queste scoperte, esiti di letture e riflessioni registrate nei primi scritti della protagonista, s'impossessa di lei una nuova forza rigenerativa dovuta anche alla maternità.

\footnotetext{
${ }^{6} \mathrm{Si}$ noti "il raddoppiamento" della personalità, la scissione dell'io che la protagonista, come tante sue amiche letterarie, che la protagonista riconosce in sé e che esprime scrivendo.
} 
La protagonista ad un certo punto rievoca uno spettacolo teatrale, un drama che la fece piangere anche se "non aveva mai pianto per le finzioni d'arte" (Aleramo 2007: 118). Si tratta della già ricordata opera di Ibsen che ella definisce un "possente genio nordico" (Aleramo 2007: 118). Le impressioni della protagonista sottolineano il momento in cui avviene in lei l'immedesimazione con Nora, "una povera bambola di sangue e di nervi che si rendeva ragione della propria inconsistenza, e si proponeva di diventar una creatura umana, partendosene dal marito e dai figli, per cui la sua presenza non era che un gioco e un diletto" (Aleramo 2007: 118). La reazione del pubblico è sorprendente per Sibilla. L'atto che Nora compie per liberarsi e ricostruirsi è vissuto con disapprovazione dal pubblico che "ammirando per tre atti, protestava con candido zelo all'ultima scena" (Aleramo 2007: 118).

Lasciare il proprio figlio anche solo in un'opera d'arte è visto come una profonda infrazione della moralità borghese. Esistono dunque due scelte possibili per la donna: quella di obbedire a questa moralità nonostante il proprio disagio o quella di ribellarsi e contemporaneamente essere isolata dagli altri e condannata alla solitudine. Proprio perché vuole dare un esempio dignitoso a suo figlio, la protagonista sceglie la seconda via, anche quando capisce che il marito non le permetterà mai di prendere con sé anche il bambino. Il momento dell'abbandono suscita in lei il desiderio di morire o di tornare nella prigione di prima, ma la voglia di vivere è più forte. Il libro che scrive diventa una specie di manifesto ma anche di un momento di confessione che dovrebbe arrivare al figlio cui vuole far capire le proprie motivazioni. E c'è di più: questo manifesto dovrebbe indurre gli uomini e le donne a comportarsi diversamente nei confronti delle proprie madri e dei propri figli per costruire un nuovo ordine sociale. L'idea e l'esempio di Sibilla Aleramo hanno suscitato, più ancora della pièce di Ibsen, uno scandalo e una serie di giudizi negativi nei confronti della scrittrice:

Mi sedevo al tavolino per scrivere delle pagine in cui rinnovavo gli appelli già lanciati alla società da ben altri ingegni, ma che io improntavo di lagrime e di sangue. I miei gridi erano ben atroci, poiché le riviste che prima mi sollecitavano, ora mi respingono (!), ma la giustizia non può essere soffocata perché arde. Io non domando fama, domando ascolto. 
E tutto si sovrappone, si confonde, e una cosa sola, su tutto splende: la pace mia interiore, la mia sensazione costante di essere nell'ordine, di potere in qualunque istante chiudere senza rimorso gli occhi per l'ultima volta.

In pace con me stessa. Spero qualcosa? No. Forse domani può giungermi una nuova ragione di esistenza, posso conoscere altri aspetti della vita, e provare l'impressione di una rinascita, d'un sorriso nuovo su tutte le cose. Ma non attendo nulla. Domani potrei anche morire... E l'ultimo spasimo di questa mia vita sarà stato quello di scrivere queste pagine.

Per lui.

Ed è per questo che scrissi. Le mie parole lo raggiungeranno. (Aleramo 2007: 203)

Il giudizio degli altri pesa fortemente, la pressione pure, ma la pace interiore fa capire che la protagonista non avrebbe potuto scegliere diversamente. Assistiamo quindi al compiersi di un processo di ricostruzione dell'io, che finalmente riesce a sentirsi libero dalle costrizioni sociali. È un io equilibrato, in armonia con il mondo. Anche se sente aprirsi in sé "una ferita mai rimarginata perché aggravata dalla rinuncia a ogni contatto con lui così come imponeva la legge disumana di quel periodo che non riconosceva alla donna nessun diritto di possesso di beni o di cura dei figli che restavano patrimonio inalienabile dei padri" (Chemotti 2009: 47), la forza che prova in questo nuovo stato, la fa andare avanti.

\section{L'ambivalenza materna}

Il romanzo e l'esperienza personale di Sibilla Aleramo sono un esempio davvero unico nella letteratura italiana. Il suo concetto della maternità fortemente originale rieccheggerà anni dopo negli scritti di Simone de Beauvoir. Nel Secondo sesso, pubblicato nel 1949, tra vari temi legati all'esistenza femminile, Simone de Beauvoir individua la maternità come la fonte sbagliata della formazione dell'identità delle donne che, una volta diventate madri, si credono realizzate: 
La fusione cercata nelle braccia del maschio e che è rifiutata non appena accordata, è realizzata dalla madre quando sente il bambino nel suo ventre pesante o quando lo preme contro i seni gonfi. Non è più un oggetto sottomesso a un soggetto; non è più un soggetto angosciato per la sua libertà, è questa realtà equivoca: la vita. Il suo corpo è finalmente suo perché è del bambino che le appartiene. La società gliene riconosce il possesso e lo riveste inoltre di un carattere sacro [...].

Alienata nel corpo e nella sua dignità sociale, la madre ha l'illusione pacificante di sentirsi un essere in sé, un valore.

Ma è solo un'illusione. Perché ella non fa veramente il figlio: questo si fa in lei; la sua carne genera soltanto dalla carne: è incapace di fondare un'esistenza che dovrà fondarsi da sola; le creazioni che emanano dalla libertà pongono l'oggetto come valore e lo rivestono di una necessità: nel seno materno, il bambino è ingiustificato, non è una proliferazione gratuita, una cosa greggia la cui contingenza è simmetrica a quella della morte. La madre può avere le sue ragioni di volere un figlio, ma non potrà dare a questo altro che esisterà domani le proprie ragioni di essere; lo genera nella generalità del suo corpo, non nella singolarità della sua esistenza. (de Beauvoir 2008: 494)

Per accorgersene, correggerla e vivere fino in fondo la propria scelta, Sibilla Aleramo ha avuto bisogno di un enorme coraggio. La sua voce letteraria si diffondeva tra chi la sosteneva e tra chi la considerava una madre snaturata. Mentre all'epoca in cui uscì il romanzo di Aleramo, la sua esperienza era considerata pioniera, il Novecento italiano è stato testimone del lavoro teorico svolto sul tema del male materno, dei lati oscuri, ambigui e ben nascosti della maternità ${ }^{7}$. Il primo passo dopo l'iniziale distacco dalle madri degli anni '70 è stato quello di amare la madre ${ }^{8}$ e accettarne l'ordine simbolico. L'ulteriore passo, svolto dalle teoriche legate al gruppo Diotima è stato quello di esplorare l'ombra della madre, cioè "affrontare l'oscuro materno come ciò che crea un cono d'ombra in cui c'è confusione tra l'io e il tu" (Gandini Baraldi 2007: 78). Il nuovo ordine simbolico a cui invitano le teoriche legate al gruppo Diotima è in contrapposizione con l'ordine patriarcale e invita le donne ad accettare la madre, amarla dal punto di vista simbolico e reale, ma anche di narrare la propria maternità e il proprio rapporto con la madre. Per compiere questo atto le madri hanno bisogno di prendere distanza dalle figlie/dai figli, per appropriarsi della propria identità e soprattutto per la paura di una

\footnotetext{
${ }^{7}$ Per un ulteriore approfondimento vedere: Diotima, L'ombra della madre.

${ }^{8}$ Luisa Muraro nel libro fondamentale L'ordine simbolico delle madri sostiene che solo la dimostrazione di gratitudine verso la donna che le ha messe al mondo, può dare alle donne un autentico senso di sé.
} 
madre onnipotente che ogni madre porta dentro di sé e che finisce per inglobare i figli se non si tiene sotto controllo. Affrontare l'oscuro materno e creare con esso l'alleanza significa esplorare anche la creatività della figlia. ${ }^{9}$

Nella letteratura degli ultimi anni si avvertono le conseguenze di quest'apertura insieme a tutte le sue contraddizioni che comporta. Le scrittrici analizzano il complesso rapporto delle donne con il materno, ma cercano anche di ridare alle madri quella possibilità di esprimersi di cui sono state private. Anche se ancora non possiamo parlare della voce delle madri, sono piuttosto le figlie a raccontarne le storie- la varietà delle sfaccettature del materno si è estesa. L'intera opera letteraria di Elsa Morante si basa sull'oscuro materno, osservato da un punto di vista maschile che priva la donna della sua identità e della sua dignità per aver infranto le leggi patriarcali. Ricordiamo un esempio offerto dal racconto di Morante, Lo scialle andaluso in cui la protagonista, la madre, lascia i propri figli per dedicarsi alla danza. Il suo tentativo di realizzarsi crolla in una performance derisa dal pubblico, osservata dagli occhi di suo figlio che giudica la madre e non riesce a perdonarle il desiderio di affermarsi in un altro modo che nella maternità. Tra tante madri "snaturate" ricordiamo la protagonista del romanzo Come prima delle madri di Simona Vinci, che deve molto al lavoro letterario di Elsa Morante, ma anche l'esempio di Lalla Romano. Si tratta del romanzo Le parole tra noi leggere, un libro autobiografico in cui la scrittrice sottopone a vivisezione il proprio rapporto con il figlio, ma anche con la maternità che la scrittrice rifiuta di vedere come espressione di sacrifio da parte della madre.

Tuttavia, le scrittrici che cercano di cambiare il modo di vivere la maternità sono ancora poche. Ancora oggi, dopo che è stato scritto molto sull'argomento, esiste un certo timore nell'affrontare l'oscuro materno e perfino nell'ammetterlo. Nonostante il concetto dell'ambivalenza materna sia ben radicato nella psicoanalisi, le aspettative culturali presumono e richiedono alla madre di amare il proprio figlio in maniere incondizionata.

\footnotetext{
${ }^{9} \mathrm{Nel}$ articolo In gioco di Wanda Tommasi Turrini, nell'edizione di Diotima, L'ombra della madre, la studiosa analizza il rapporto di Marguerite Duras con la follia di sua madre: la straordinaria forza della madre si traduce in vena creativa di Duras. L'alleanza con l'oscuro materno fa nascere la scrittura in cui viene data la voce alla madre.
} 
Nonostante i problemi legati al tentativo di affrontare il male materno, la studiosa Marianne Hirsch nel libro The mother/daughter plot. Narrative, Psychoanalysis, Feminism insiste affinché le donne continuino a mettere in discussione l'errata visione del materno presente nella società che vede nella madre una figura del sacrificio. La studiosa sottolinea che nei testi letterari che raccontano il rapporto materno è importante far parlare le madri, perché "feminist analysis is still written from the child's primary process perspective: permeated with desires for the mother's approval, with fear of her power, and with anger and resentment at her powerlessness" (Hirsch 1989: 169).

\section{La situazione attuale in Italia}

Anche la letteratura italiana dimostra negli ultimi decenni un'apertura verso la voce materna che, a sua volta, racconta questo rapporto non più in maniera idealizzata, ma conflittuale e ambigua; tuttavia, la maggioranza dei testi è ancora scritta dal punto di vista della figlia che tende o a idealizzare la madre o ad annientarla.

Quando le scrittrici italiane trattano l'ambivalenza materna, le deviazioni nel comportamento delle madri e delle figlie, parlano di amore e di odio, delle frustrazioni mischiate con la tenerezza, seguendo l'esempio di teoriche come Adrienne Rich, una di tante donne che ha trovato il coraggio di dare voce all'ambiguità del materno, parlando della propria esperienza del materno e decostruendone la glorificazione:

I miei figli mi danno le più squisite sofferenze che mai abbia conosciuto. È la tortura dell'ambivalenza: il dilaniante alternarsi di amaro risentimento ed esasperazione, e gioiosa gratificazione e tenerezza. A volte, nei miei sentimenti verso questi piccoli esseri innocenti, mi vedo come un mostro di egoismo e di intolleranza. Le loro voci mi logorano i nervi, le loro continue necessità, soprattutto il loro bisogno di semplicità e di pazienza, mi riempiono di disperazione per le mie inadeguatezze, e anche per il mio destino che mi impone di assolvere un compito per il quale non sono adatta. E talvolta mi sento sfibrata dalla rabbia soffocata. Ci sono momenti in cui ho l'impressione che solo la morte ci libererà l'uno dall'altro, momenti in cui invidio la donna sterile che si può permettere il lusso dei rimpianti ma vive una vita autonoma e libera. (Rich 1977: 19) 
Raccontando i propri disagi legati alla maternità, Rich invita le donne ad esprimersi, a raccontarsi e a parlare soprattutto della rabbia soffocata perché essa "becomes a dominant theme in exploration of maternal subjectivity" (Hirsch 1989: 169). Per arrivare alla costruzione della soggettività materna, bisogna affrontare violenza nascosta, ma anche quello che viene chiamato la "prepotenza della funzione materna" (Ferrante 2003: 290). Questo termine significa che la donna, una volta diventata madre, è legata per sempre al figlio, nonostante le altre funzioni che possa svolgere nella società. Si trova "intrappolata" nel suo istinto materno e questo fatto non dipende soltanto dalla sua condizione sociale o dal suo rapporto con il padre del figlio, ma è una condizione interiore da cui, come dice Rich solo la morte potrà liberarla. Questa condizione intima e spaventosa è descritta nella sua essenza da Natalia Ginzburg:

Il femminismo afferma che i lavori di casa, e la cura dei figli, dovrebbero essere equamente divisi fra donne e uomini. Questo, come ogni altra richiesta pratica e concreta dei movimenti femminili, mi sembra giustizia. Nel femminismo esiste però l'idea falsa che i lavori di casa, e la cura dei figli, siano un'umiliazione. Non è vero che i lavori di casa, e la cura dei figli, debbano essere divisi con gli uomini perché umilianti. Devono essere divisi con gli uomini perché, fra uomo e donna, tutto dovrebbe essere equamente diviso, come tutto deve essere diviso fra uguali.

Poiché sono le donne a generare i figli, il peso di accudirli e crescerli tocca soprattutto alle donne. Esiste fra le madri e i figli un rapporto a cui non si sfugge perché attraversa e confonde insieme le vie delle viscere e le vie dello spirito. Una donna all'uomo può chiedere aiuto, ma il peso dei figli rimane comunque sulle sue spalle. Essa si sente tenuta ad accudire ai figli. Quando non lo fa, si sente in colpa, e quando lo fa, si sente ansiosa e irrequieta e traduce allora l'ansietà e l'irrequietudine in un senso di umiliazione e frustrazione. Le sembra che non avrà mai più pace e non sarà mai più libera. Si dibatte nei grovigli dell'affetto, come una bestia presa in trappola, e poiché l'affetto materno è un sentimento che non rassomiglia a nessun altro, i legami oscuri e viscerali che tengono una donna stretta al figlio le sembrano il contrario della chiarezza e della libertà. Ma contro una simile sensazione, non esistono difese possibili, essendo una sensazione d'angoscia che non ha nulla da fare con le colpe della società. Un errore è credere che una simile sensazione, essendo oscura e viscerale, sia umiliante. Come la condizione femminile, così anche la maternità non è in se stessa né una ragione d'umiliazione, né una ragione d'orgoglio. Come la condizione femminile, non è nulla in se stessa. Essenziale è soltanto riconoscerne e amarne insieme la felicità e il dolore, essendo negli affetti la felicità e il dolore indivisibili l'uno dall'altra. (Ginzburg 1987: 651-652) 
Notiamo che Natalia Ginzburg è una delle prime donne che abbiano definito il rapporto con il figlio come "un rapporto che confonde le vie delle viscere e le vie dello spirito". Vi leggiamo ciò che Elena Ferrante tanti anni dopo chiamerà la già menzionata "prepotenza della funzione materna".

\section{La figlia oscura di Elena Ferrante}

Un esempio letterario che sembra incarnare quanto detto nel paragafo precedente è il romanzo della stessa Ferrante, La figlia oscura, pubblicato nel 2006. Quest'opera segue il processo d'introspezione dell'io narrante Leda, una madre di due figlie che cerca di venire a capo del suo rapporto complicato e ambiguo con le figlie e con la propria madre, ma prima di tutto con se stessa. Le figlie sono ormai cresciute e si sono trasferite dal padre per intraprendere una carriera universitaria. Leda parte per una vacanza che diventa il viaggio d'introspezione e del confrontarsi con i propri sensi di colpa e gli altri modelli della maternità incarnati nei personaggi femminili incontrati in spiaggia. I sensi di colpa rotolano intorno all'abbandono delle figlie, ricordato dall'io-narrante, quando le figlie erano ancora piccole e concluso tre anni dopo, quando Leda torna ad occuparsi delle figlie. Rispetto al romanzo di Sibilla Aleramo, possiamo trovare dei punti in comune. In entrambi i casi si tratta di madri giovani, che si sentono non realizzate e piene di angoscia esistenziale, di donne a cui manca lo spazio per esprimersi. Le condizioni sociali sembrano essere però completamente diverse.

Mentre la protagonista di Una donna deve lasciare il figlio al marito, perché all'epoca tutti i diritti appartenevano al padre, Leda, l'io narrante del romanzo è una donna che lavora all'università, che ha la libertà legale di lasciare le figlie con il marito e di tornare a prenderle. Il diritto al lavoro e alla realizzazione personale è acquisito. Tuttavia, questa uguaglianza è solo apparente perché Leda si trova socialmente e intellettualmente isolata nel ruolo di madre. Mentre si occupa di figlie e di casa, cerca di studiare e scrivere invano. L'angoscia e la rabbia crescienti la portano a compiere il distacco e quello che 
inizia come un viaggio breve per partecipare a un convegno in Canada, diventa un soggiorno lungo tre anni.

La protagonista di Una donna se ne va per dare un esempio non solo al figlio, ma anche ad altre persone in cerca della libertà dello spirito. La sua lotta consiste nel tentativo di migliorare la società, di far diventare le madri non più povere creature di sacrificio, ma individui veri che decidono da soli il proprio modo di crescere e di essere. La protagonista di La figlia oscura, Leda, non ha questo dovere terrificante addosso, ma agisce secondo la sua voglia individuale, in possesso di quella libertà a cui Sibilla aspirava. Anche lei, e siamo stavolta all'inizio del XXI secolo, deve affrontare l'incomprensione e i pregiudizi degli altri, incarnati però da persone a lei estranee, e non dalla sua famiglia che l'appoggia per quanto sia possibile date le circostanze. Leda va in Canada perché vuole finalmente "realizzarsi", appropriarsi della lingua che insegna, studiare, scrivere, esplorare la propria vena creativa. La maternità in lei dopo l'iniziale annullamento della donna diventa l'esplorazione dei propri limiti e potenzialità. Nella sua assenza, è il marito ad occuparsi delle figlie. Il rapporto tra i due coniugi crolla, ma la comprensione della famiglia non viene meno. Il viaggio introspettivo di Leda comincia a distanza di venticinque anni dopo il ritorno alle figlie, nel momento in cui si trova da sola in vacanza e conosce Nina, giovane madre napoletana, accompagnata dalla figlia e dalla rumorosa famiglia del marito. Simbolicamente, l'incontro che avviene tra le due donne provenienti dallo stesso background, cioè la città di Napoli, ma che hanno intrapreso due strade diverse di formazione, Leda l'indipendenza e Nina la dipendenza dal marito e la maternità, porta alla presa di coscienza di entrambe. Leda, incatenata dall'oscuro materno in sé riesce a liberarsi dei propri sensi di colpa solo mettendosi di fronte alla ragazza, narrando la propria esperienza. La ragazza, a sua volta, ascoltando la narrazione di Leda, giunge all'accettazione dell'angoscia costante che le provoca la maternità, la sensazione quando "ti si sfrantuma il cuore: non riesci a sopportare di stare insieme a te stessa e hai certi pensieri che non puoi dire" (Ferrante 2006: 127). La condizione di Nina ricorda quella della protagonista del romanzo Una donna prima della scoperta dei libri e delle letture che l'hanno resa più forte. Anche se non ne è cosciente, anche a lei è data la possibilità di aprire i propri orizzonti. Il contatto con Leda, donna in carriera che ha avuto il coraggio 
di lasciare le proprie figlie per tre anni, tutte cose che in una ragazza semplice suscitano ammirazione e rispetto, muovono qualcosa dentro di Nina. L'importanza di questo contatto non sta nelle conseguenze dirette dell'incontro tra due mondi femminili opposti, quanto nel fatto che le due donne possono parlare liberamente della loro condizione. Solo affrontando le proprie angosce, si arriva alla liberazione. Il tabù viene infranto e così si oltrepassa un varco sociale, individuale ed emotivo. Proprio questa libertà di agire, di cercarsi e di permettersi la libertà di esplorare le proprie potenzialità creative, permette a Leda di scegliere una via di mezzo, un compromesso tra se stessa, la carriera e la famiglia:

Tornare a casa, dalle figlie, per Leda significa mettere al centro della sua ricerca non il puro e semplice fatto di averle partorite, ma la pienezza della maternità. Prima, con la fuga, ha cercato un'emancipazione e un confronto paritario col mondo maschile. Dopo, col ritorno, la sua vita pubblica, il lavoro, i pensieri, gli amori fanno centro su quella che io definirei la prepotenza della funzione materna. (Ferrante 2003: 290)

Elena Ferrante esplora proprio questi punti deboli del materno nel momento in cui le vecchie costrizioni sociali sono ormai il passato e la donna è libera per quanto sia possibile di scegliere la propria strada. Il ritorno alle figlie significa accettare la maternità ma non annientarsi. Alla domanda di Nina, su quale sia il possibile modo di vivere la maternità, Leda risponde che ha imparato a "vivere poco per se e molto per le due bambine" (Ferrante 2006: 117).

Quello che i romanzi di Elena Ferrante mettono in discussione è la figura materna con, appunto, i propri bisogni che vengono soffocati nella maternità così come è concepita dall'ordine patriarcale. Un altro personaggio ferrantiano che mette in discussione il ruolo della donna è quello di Olga, ne I giorni dell'abbandono. Si tratta di un romanzo in cui viene affrontato il percorso interiore di una donna, madre di due figli, che è stata abbandonata dal marito. Anche in questo romanzo è analizzato "che dopo essersi annientata nella madre/moglie, si ritrova sola e confrontata al "vuoto di senso"” (Marchais 2006: 56): "Non avevo più lavoro, nessun lavoro, nemmeno lo scrivere, da almeno cinque 
anni. [...] Facevo la spesa, cucinavo, rassettavo, mi trascinavo i due bambini di via, in via, di stanza in stanza, stremata, esasperata" (Ferrante 2002: 101).

Olga cade in depressione, in uno stato di completa perdita di identità. Capisce che deve combattere le imposizioni sociali che vedono in lei una madre e non più una donna sessuata. La presenza dei bambini non riesce a riempire il vuoto esistenziale. I figli rappresentano solo l'ostacolo che si crea tra lei e il marito, perché la deprivano della femminilità. È tormentata dal ricordo di una donna napoletana chiamata "poverella":

L'odore acido del vomito mi ricordò il tempo delle poppate, delle pappine, i rigurgiti improvvisi. Pensai, mentre cancellavo da pavimento con mosse lente la traccia del malessere di mio figlio, alla donna di Napoli con quei suoi bambini lagnosi, tacitati a forza di caramelle. Da un certo punto in poi lei, la moglie abbandonata, aveva cominciato a prenderesela con loro. Diceva che le avevano lasciato l'odore di mamma addosso, e questo l'aveva rovinata, colpa loro se il marito se n'era andato. Prima ti gonfiano la pancia, prima ti appessantiscono le mammelle, e poi non hanno pazienza. Parole così mi ricordai. Mia madre le ripeteva a bassa voce per non farmele sentire, gravemente, consentendo (Ferrante 2002: 101).

Olga come Leda capirà che i figli hanno bisogno di lei e che per essere buona madre, la donna deve costruire se stessa come un soggetto, deve rispettare la propria essenza. Il percorso che fa per riconquistare la propria identità persa l'aiuta a diventare una madre serena e piena d'affetto.

\section{Conclusioni}

I romanzi di Elena Ferrante invitano a "riflettere anche su una società, la nostra, che pur riconoscendo alle donne autonomia e uguaglianza, quantomeno formale, non permette loro di conciliare vita professionale e vita familiare, di far coesistere la donna e la madre" (Marchais 2006: 54). 
Questi romanzi "riconoscono la violenza della maternità patriarcale" (Rich 1977: 266) e finalmente affrontano la figura materna con tutte le sue ambiguità e ombre. La condizione delle donne sembra ancora riconducibile a "un ruolo fisso e delimitato, in cui l'identità materna finisce per inglobare le altre" (Marchais 2006: 57). Perciò la richiesta di Sibilla Aleramo, il suo postulato di cercare in ogni madre una donna, risulta attuale nonostante i cambiamenti sociali avvenuti nell'arco di un secolo. Il dialogo intertestuale che ho cercato di stabilire tra i testi di Aleramo e Ferrante, continua a guadagnare nuove/i parlanti nelle voci narranti che raccontano l'esperienza della maternità. Queste voci rifiutano nella maternità esclusivamente il sacrificio, esplorano fino in fondo il male oscuro della maternità e ne trovano potenzialità creative sempre più potenti, in grado di rompere $\mathrm{i}$ tabù e di creare una società più umana.

\section{Riferimenti bibliografici}

\section{Bibliografia primaria}

Aleramo, Sibilla. 2007. Una donna. Milano: Feltrinelli.

Ferrante, Elena. 2002. I giorni dell'abbandono. Roma: Edizioni elo. 2006. La figlia oscura. Roma: Edizioni elo.

Ginzburg, Natalia. 1987. Opere, 2 vol. Milano: Mondadori.

Ibsen, Henrik. 2002. La casa di bambola. Milano: Mondadori.

Morante, Elsa. 2001. Opere. Milano: Mondadori.

Romano, Lalla 1996. Le parole tra noi leggere. Torino: Einaudi. 


\section{Bibliografia secondaria}

Chemotti, Saveria. 2009. L'inchiostro bianco. Madri e figlie nella narrativa italiana contemporanea. Padova: Il Poligrafo.

De Beauvoir, Simone. 2008. Il secondo sesso. Milano: Il Saggiatore.

Ferrante, Elena 2003. La frantumaglia. Roma: Edizioni elo.

Gandini Baraldi, Sara 2007. "La luna e le lunine". L'ombra della madre. Eds. Diotima. Napoli: Linguori. 77-85.

Hirsch, Marianne. 1989. The mother/daughter plot. Narrative, Psychoanalysis, Feminism. Indianapolis: Indiana University Press.

Marchais, Nathalie. 2006. "Madre o donna? Figure femminili in conflitto nei romanzi di Elena Ferrante". Écritures, 2: 45-59.

Rich, Adrienne. 1977. Nato di donna. Milano: Garzanti.

Tommasi Turrini, Wanda 2007. “In gioco". L'ombra della madre. Napoli: Diotima. 163175. 